\title{
Astrobiological Humanities Group for Interplanetary Protection and Defense Policies
}

\author{
Author \\ FLORA ARDENGHI DUTRA \\ floradutra@gmail.com \\ Federal University of Rio Grande do Sul \\ Graduate Department in Social Anthropology
}

Flora A. Dutra

+55 (55) 98445-7911

floradutra.com

Submitted to:

Planetary Science and Astrobiology Decadal Survey 2023-2032.

The National Academy of Sciences, Engineering and Medicine. 


\section{Introduction - Humanities' intrinsic relationship with Space Policies}

To start the discussion on the role of space researchers and their responsibilities with the Planetary Protection and Defense Policies, we must cover other celestial bodies, which is why they must be thought within an interplanetary context in the Planetary Science and Astrobiology Decadal Survey 2023-2032. Scientific investigations looking for ways to detect life in our Solar System should not be compromised by the lack of Policies that protect from the potential risk of contact and interplanetary biological cross-contamination. But it is not only in the field of biological contamination of astronauts and celestial bodies that the Interplanetary Protection and Defense Policies must be activated. The latest revisions requested by the National Academy of Sciences, Engineering, and Medicine sparked debates about the representativeness of Committee on Space Research (COSPAR), NASA Policies, and the emergence of society's involvement. An independent committee was created revealing discrepancies to be adjusted to the latest version of the document released by COSPAR based on Article IX of The Outer Space Treaty.

The latest review by the Committee on Space Research (COSPAR), published in June $2020^{1}$, fails to communicate to global society about changes in its policies, which alter, restrict and release processes and protocols without a broad dialogue with society and the strong commitment to scientific communication or public education highlighting its impacts and consequences. It is necessary to incorporate in the policies that deal with the next decade of space exploration, a language that crosses the Humanities so that it contemplates all sectors of society and its diverse audiences. NASA's advisory councils evaluate and review the development of these policies that are maintained by COSPAR, these investigations must go in the same advance as space technology through Interplanetary Protection and Defense Policies and their relationship with society. All major space-traveling nations, since the beginning of the Space Age, have tried to minimize cross-biological contamination.

Because of these concerns, it is up to the community of researchers that make up the Astrobiology network for the next decade to accept implementing the interdisciplinarity of scientific fields, filling an urgent gap that belongs to the Humanities in the development of Interplanetary Protection and Defense Policies. The conflicts of interest that permeate academic fields must be overcome by a greater insertion of Astrobiology in global society. The proposal sent here seeks to encourage the involvement of strategies and guidelines both from the National Academy of Sciences, Engineering and Medicine, as well as from space agencies and civil society with a special attention to the private sector's commitment to the ethical compliance with the Interplanetary Protection and Defense Policies. We must encourage the involvement of diverse scientific communities as global organizations that have representation in different cultures and peoples, assuming an ethical commitment to the search for life in interplanetary missions.

\footnotetext{
${ }^{1}$ COSPAR Policy on Planetary Protection $\rightarrow$ https://cosparhq.cnes.fr/assets/uploads/2020/07/PPPolicyJune-2020 Final Web.pdf
} 


\section{The inconsistencies of the Planetary Defense and Protection Policy Reports}

With increasingly frequent launch missions, the Space Studies Board (SSB) of the National Academies is part of the history of the Space Age, acting for more than 60 years in the Planetary Protection Policy Reports. Developments in these study centers included policy planning for the development of important missions involving Astrobiology and new international space agencies. In 2019, NASA established a Planetary Protection Independent Board Recommendations $(\mathrm{PPIRB})^{2}$ to assess the agency's policies in this area. The evaluation of the Space Studies Board (SSB) contains 77 conclusions divided into three categories: a) consistent reports; b) inconsistent reports c) comparable elements between reports. In the general comment on the area of consistency between the reports from NASA, COSPAR, SSB and the National Academies is the agreement that Planetary Protection Policies are equally applicable to governmental and nongovernmental missions. International cooperation also stands out as the point that the private space exploration sector changes Planetary Protection and Defense Policies.

We believe that in the next few years there will be a reorganization of the Planetary Protection and Defense Office as well as the modification of clear public communication on Space Policies. Below, we address the inconsistencies, risks, concerns and non-comparable areas highlighted by the Planetary Protection Independent Board Recommendations (PPIRB):

a) Update the representativeness of the functions of COSPAR and The United Nation (UN) for the peaceful use of outer space and its development of Planetary Policies;

b) The term Planetary Protection is still confusing and must dialogue with the relevance and obligations of The Outer Space Treaty;

c) Reclassification of spacecraft missions to the Moon, Mars and other bodies of ts.the Solar System as well as NASA opportunities to provide Planetary Protection assistance to the private sectors of space exploration. It will also be possible to issue sanctions to the private sector that does not comply with the determined responsibilities and obligations.

In suggesting to establish a new Advisory process clarifying legal and regulatory issues, new scientific and technical bases emerge from a consultative process for Planetary Protection Policies that must be reevaluated due to the rapid advance of scientific, technological and planetary mission capabilities. For this reason, it is recommended to establish an emerging permanent forum that includes contributions from the government, the private sector and international cooperation. When requesting the establishment of an independent and appropriate advisory body to assist in the formulation and implementation of Planetary Policies, the Humanities were never mentioned as a science complementary to the multiplicities of the technical staff and consultants.

\footnotetext{
${ }^{2}$ NASA Response to Planetary Protection Independent Review Board Recommendations https://www.nasa.gov/sites/default/files/atoms/files/planetary protection board report 20191018.pdf
} 


\section{Responsible Guidelines from the Humanities for Interplanetary Protection and Defense Policies}

It is fundamental and essential that for the next ten years, the official documents foresee the removal of the space debris that orbit the planet Earth with notes for solutions that do not cause irreversible changes in the environment of outer space when removing these space debris. Researchers, space agencies, governments, the private sector and the public must be aware of the irreversible changes both in low orbit and in outer space caused by responsible policy failures and lack of ethics with the scientific community causing harm to humanity. Emerging laws must rise to give consensus on security and low risk implementations. Social Policies that contemplate the understanding of the exploration and astrobiological contamination of the Moon, Mars or Venus are important in the regulation that must also be extended outside the Solar System maintaining universally accepted guidelines for the protection of all. An interdisciplinary dialogue across the Humanities must be incorporated into specialized bodies such as the UN, COSPAR, the National Academies, NASA Planetary Protection Office and other space agencies. We suggest, below, some global social guidelines required by the Humanities related to the responsibility of Interplanetary Protection and Defense Policies that should dialogue with the global society for the next years.

Global social Responsibilities for Protection Policies and Interplanetary Defense.

a. Removal of the active space debris that orbits the planet as a preventive measure to change the Earth's lower atmosphere forever. Space pollution must be controlled, regulated and removed to protect new launches and prevent future catastrophic collisions between launched objects, astronauts and the return and fall of these artifacts to the Earth's surface.

b. Manage and implement social requirements that guide our society towards a greater understanding of political standards that govern the Protection and Defense of Earth, astronauts and other celestial bodies.

c. Develop new social standards and universal policies that include experiences and research projects in the Humanities area as a technical-based organization service specialized in Interplanetary Protection.

d. Define social requirements and protocols to guarantee the support of the Humanities in the global debate on Interplanetary Protection and Defense Policies. Organize, teach and disseminate the Interplanetary Protection and Defense Policies to future generations based on the scientific communication of the Astrobiological Humanities Group contemplating the global society on the updates of the protocols and the Space Treaties, regulations and government policies as well as the possible global impacts of future missions for the next ten years. 


\section{Elaboration of Spatial Social Agreements and Treaties related to Humanities and Interplanetary Protection Policies}

All space-traveling nations of world governments as well as private sector companies must establish meetings, assemblies and review boards to adapt the successful consistencies of their Protection and Defense Policies. With the example of the successful review of Planetary Protection Independent Board Recommendations in early 2019 that pointed out flaws and inconsistencies, it also pointed out recommendations for the sectors involved. When drafting International Space Agreements and Treaties that also contemplate the Humanities, Ethics, commitment and responsibility towards humanity and interplanetary bodies, recommendations from global society are required. Emerging laws of Space Law and Articles of The Outer Space Treaty itself should be revised.

Social, political and ethical issues are not addressed with due consistency as technical errors and new implementations are pointed out. Despite important and crucial contributions by scientists who develop such protocols, there is an urgent need for a Humanities technical team to better incorporate and transmit knowledge from generation to generation. The Interplanetary Protection and Defense Policies must dialogue with the intelligence and human collectivity, as we will be the ones to explore other possible worlds in the coming years.

\section{Social elements and actions as spatial policy guidelines for the Humanities}

a. Develop a strategic action plan that includes increasing the participation of public education and future generations supporting gender equality and diversity in the creation and expansion of new space policies.

b. Adjust technical journals with a language and vocabulary accessible to the global society to reach the public and give visibility and importance to the updates and constant changes in the Interplanetary Protection and Defense Policies.

c. Carry out strategic actions of comprehensive space policies so that the Planetary Science and Astrobiology Decadal Survey 2023-2032 with global influence through scientific leaders recognized by the world community create engagement with the Social and Human Sciences to fulfill the responsibilities and principles of the Interplanetary Protection and Defense Policies.

d. Include society, public education and scientific communication in the formation and design of new Interplanetary Policies, expanding with the Humanities the scope of new information incorporated into the International Space Treaties and Agreements. Join international efforts and contemplate global rules for space exploration using space resources peacefully. Even with less stringent restrictions, the space environment should always be a mediator for interplanetary sustainable development in the coming years.

e. A social inventory of Interplanetary Protection and Defense Policies with qualitative descriptions should be mapped in order to include interdisciplinary areas of the Humanities such as Law, Political Science, Psychology, Philosophy, Anthropology, Ethics, etc. 


\section{Astrobiological Humanities Group for the Global Social Scientific Council and Interplanetary Space Ethics}

The objective of the creation of the Astrobiological Group of Humanities is to assist in the creation of the Global Social Scientific Council and Interplanetary Space Ethics, which will be dedicated to international cooperation in space policy research and its dialogical improvements with society, sharing results and scientific collaborations in the Social Sciences, links with future generations, public education and scientific communication.

The Astrobiological Humanities Group will be able to collaborate actively in the Interplanetary Protection and Defense Policies from different social perspectives and the Interdisciplinarity of the Humanities. The objective is to present to society comments and information that will help the National Academies in the relationship with the general public and scientific communication.

Activities and objectives of the Global Social Scientific Council and Interplanetary Space Ethics

a. Encourage scientists around the world through scholarships and funding to manage future formulations of interplanetary policies by influencing space research through communication with the public and extending the benefits of space research to society.

b. Unify international laws for the commercial, state and private use of interplanetary outer space through the ethical foundations of Space Law and legislation for all nations.

c. Establish rules with nations for the properties of metals, minerals and other resources found in celestial bodies.

d. The amount of space debris must be reviewed with society, as space waste may compromise future missions and its consequences must be communicated to society as a matter of urgency. Regardless of economic and scientific development, the revision of Article IX of the Outer Space Treaty must be done by all nations encouraged by the Planetary Science and Astrobiology Decadal Survey in partnership with the UN.

e. The activities of the Group and the Council should promote space policies at the international level with an emphasis on public debate open to all scientists in the Social and Human Sciences, guaranteeing security in the Geopolitical and Biopolitical dialogue, without conflicts or social and technological differences between nations. 


\section{Final considerations}

Humanity's next challenge in the coming years will be the ethics and boundaries of space exploration. This process must be widely communicated to the global society in questions that determine what can be authorized or not. One of our recommendations will be the balance between the Pure Sciences and the Humanities when communicating new space policies that contemplate the search for life in our Solar System and beyond. The development of new technologies in their practical applications as future missions must involve human social activities and their interdisciplinary areas in a joint effort with the scientific community in different areas. The task of communicating with society should not be the sole responsibility of COSPAR, the responsibility of all involved is beyond just one organization, office or agency, and this effort must be broad, connected, diversified and responsible for a set of leaders.

In addition to establishing a new range of relevant scientific disciplines for the Humanities in the formulation of Interplanetary Protection and Defense Policies, we recommend new mechanisms for the emergence of issues of these policies in close dialogue with society by establishing processes in compliance with the Astrobiological Humanities Group for the Council Global Social Scientific and Interplanetary Spatial Ethics based on responsible suggestions and guidelines from the Humanities for Interplanetary Protection and Defense Policies by highlighting the inconsistencies of the Planetary Protection and Defense Policy Reports.

The regulatory gaps that permeate these spatial policies must incorporate new forms of knowledge, production and formulation contemplating the interdisciplinarity that is characteristic of Astrobiology, inserting the Humanities so that they do not remain on the edge of history and aggregate what will be the best for years to come. For new future situations, such as private sector missions to other celestial bodies or the human exploration of Mars, policies and their potential impacts on humans must be evaluated and examined well in advance by the Astrobiological Humanities Group for Interplanetary Protection and Defense Policies.

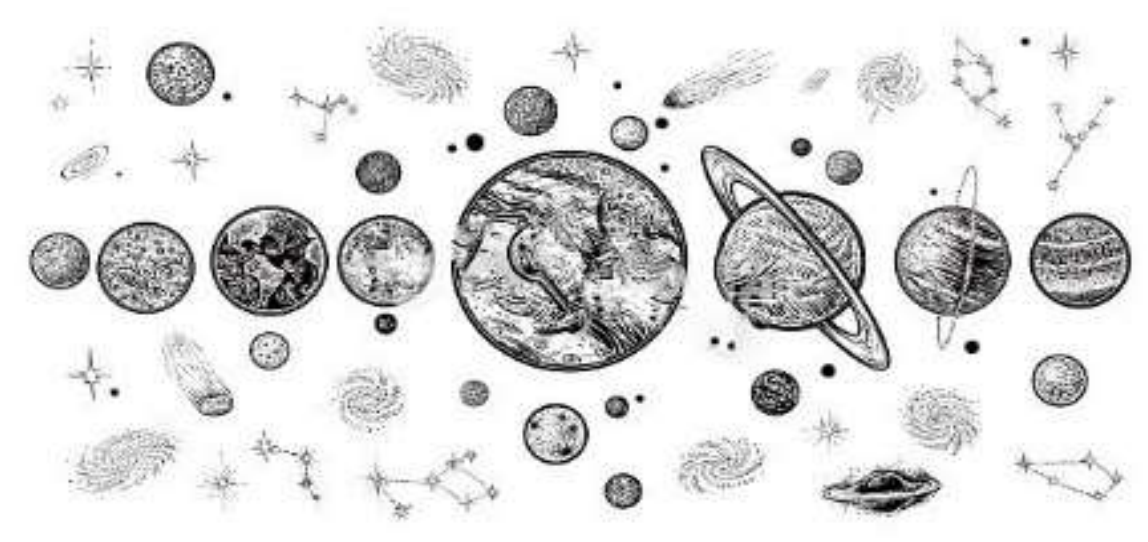




\section{References}

The National Academies of Sciences, Engineering, and Medicine 2020.

Assessment of the Report of NASA's Planetary Protection Independent Review Board. Washington, DC: The National Academies Press.

$\rightarrow$ https://doi.org/10.17226/25773.

COSPAR Policy on Planetary Protection, 2020.

$\rightarrow$ https://cosparhq.cnes.fr/assets/uploads/2020/07/PPPolicyJune-

2020 Final Web.pdf

The Office of Safety and Mission Assurance (OSMA), 2020.

$\rightarrow \underline{\text { https://sma.nasa.gov/ }}$

NASA Payload Safety Program, 2020.

$\rightarrow$ https://nodis3.gsfc.nasa.gov/displayDir.cfm?Internal ID=N PR 8715 007B

\&page name=main

Safety and Mission Assurance (SMA) Audits, Reviews and Assessments, 2019.

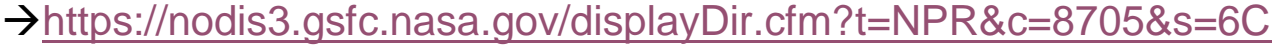

Review and Assessment of Planetary Protection Policy Development Processes, 2018.

$\rightarrow \underline{\text { https://www.nap.edu/catalog/25172/review-and-assessment-of-planetary- }}$ protection-policy-development-processes

Assessment of the Report of NASA's Planetary Protection Independent Review Board, 2020.

$\rightarrow$ https://www.nap.edu/catalog/25773/assessment-of-the-report-of-nasasplanetary-protection-independent-review-board

Planetary Protection Classification of Sample Return Missions from the Martian Moons, 2019.

$\rightarrow$ https://www.nap.edu/catalog/25357/planetary-protection-classification-ofsample-return-missions-from-the-martian-moons

Review and Assessment of Planetary Protection Policy Development Processes, 2018.

$\rightarrow$ https://www.nap.edu/catalog/25172/review-and-assessment-of-planetaryprotection-policy-development-processes

The Goals, Rationales, and Definition of Planetary Protection, 2017.

$\rightarrow$ https://www.nap.edu/catalog/24809/the-goals-rationales-and-definition-ofplanetary-protection-interim-report

NASA Response to Planetary Protection Independent Review Board Recommendations, 2019.

$\rightarrow \underline{\text { https://www.nasa.gov/sites/default/files/atoms/files/planetary protection boar }}$

d report 20191018.pdf 